\title{
ANALYTICAL EVALUATION OF THE SECOND ORDER MOMENTUM COMPACTION FACTOR AND COMPARISON WITH MAD RESULTS
}

\author{
J.P. SHAN, S.G. PEGGS ${ }^{\dagger}$ and S.A. BOGACZ \\ Fermi National Accelerator Laboratory, ${ }^{,}$, P.O. Box 500, Batavia, IL 60510
}

(Received 27 April 1992; in final form 7 March 1994)

\begin{abstract}
The second order momentum compaction factor $\alpha_{1}$ is a critical lattice parameter for transition crossing in hadron synchrotrons and for the operation of quasi-isochronous storage rings. These have been proposed for free electron lasers, synchrotron light sources and recently for high luminosity $e^{+} e^{-}$colliders. First, the relation between the momentum compaction factor and the dispersion function is established, with the "wiggling effect" included. Then, an analytical expression of $\alpha_{1}$ is derived for an ideal FODO lattice by solving the differential equation for the dispersion function. A numerical calculation using MAD is performed to show excellent agreement with the analytical result. Finally, a more realistic example, the Fermilab Main Injector, is also considered.
\end{abstract}

KEY WORD: Momentum compaction, dispersion function, particle dynamics, storage rings, synchrotrons

\section{INTRODUCTION}

In a synchrotron or storage ring, particles with different momenta have different closed orbits. The difference in the closed orbit length $(\Delta C)$ between a particle with momentum $\mathrm{p}$ and a reference particle with momentum $p_{0}$ may be expressed as an expansion in momentum offset $\delta$

$$
\Delta C=C_{0} \alpha_{0} \delta\left[1+\alpha_{1} \delta+O\left(\delta^{2}\right)\right]
$$

where $C_{0}$ is the length of the reference orbit, and

$$
\delta=\frac{p-p_{0}}{p_{0}}=\frac{\Delta p}{p_{0}}
$$

\footnotetext{
† Now at Brookhaven National Laboratory, Upton, NY 11973.

* Operated by the Universities Research Association Inc., under contract with the U.S. Department of Energy.
} 
A dependence of orbit length on momentum is called momentum compaction, and $\alpha_{0}$ is the linear momentum compaction factor. The second order momentum compaction factor, $\alpha_{1}$, is the focus of this paper.

Although rooted in the transverse motion, the momentum compaction effect influences the longitudinal motion through the phase slip factor,

$$
\eta=\frac{1}{T_{0}} \frac{T-T_{0}}{\delta}=\eta_{0}+\eta_{1} \delta+O\left(\delta^{2}\right)
$$

where

$$
\begin{aligned}
& \eta_{0}=\alpha_{0}-\frac{1}{\gamma^{2}} \equiv \frac{1}{\gamma_{T}^{2}}-\frac{1}{\gamma^{2}}, \\
& \eta_{1}=\alpha_{0} \alpha_{1}+\frac{3 \beta^{2}}{2 \gamma^{2}}-\frac{\eta_{0}}{\gamma^{2}} .
\end{aligned}
$$

Here $T$ is the period of revolution for a particle with momentum offset $\delta$ and $T_{0}$ is for a synchronous particle, $\beta$ and $\gamma$ follow usual relativistic kinematic notation, and $\gamma_{T}$ is the transition gamma for a synchronous particle. For a conventional FODO lattice, $\gamma_{T}$, is roughly equal to the horizontal tune of the machine, which is about $5 \sim 30$ depending on the size of machine. Therefore, most of the medium energy hadron synchrotrons have to cross transition. Since $\delta \ll 1, \eta_{0}$ is the dominant contribution to $\eta$ except near transition where $\eta_{0}$ is very small and the first nonlinear term

$$
\eta_{1} \approx \alpha_{0}\left(\alpha_{1}+\frac{3}{2}\right)
$$

becomes very important, while the contribution from even higher order nonlinear terms can still be neglected. Nonzero $\eta_{1}$ leads to the fact that higher momentum particles and lower momentum particles can not agree when the synchronous phase should be switched. ${ }^{1}$ In reality, the phase switch can only happen at one particular instant, so most particles have to experience a defocusing radio frequency (rf) force for a period of time. This may be the dominant mechanism causing emittance blowup and possibly beam loss for some machines, according to tracking studies ${ }^{2-4}$ and a preliminary experiment. ${ }^{5}$ If we can set $\alpha_{1}=-1.5$, the nonlinear effect will be suppressed and transition crossing will become much less harmful.

All electron machines operate well above transition, or $\gamma \gg \gamma_{T}$; therefore

$$
\eta_{0} \approx \alpha_{0}, \quad \eta_{1} \approx \alpha_{0} \alpha_{1} .
$$

While the bunch length is roughly proportional to $\sqrt{\eta_{0}}$, it may be possible to get very short bunches by using quasi-isochronous rings with very small $\alpha_{0}$ values. These have been proposed for free electron laser drivers, ${ }^{6}$ for synchrotron light sources ${ }^{7}$ and for the next generation of high luminosity $e^{+} e^{-}$colliders. ${ }^{8}$ There, the momentum acceptance of the rf bucket, which goes roughly like $\eta_{0} / \eta_{1}\left(\right.$ or $\left.\alpha_{1}^{-1}\right),{ }^{8,9}$ should be larger than 10 times the root-mean-square (rms) momentum spread of bunch $\delta_{r m s}$ in order 


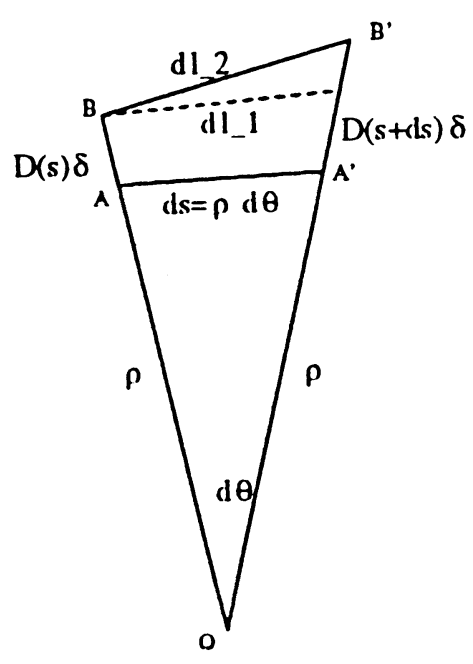

FIGURE 1: Schematic view of the wiggling effect

to preserve reasonable quantum life time. That is,

$$
\alpha_{1}<\frac{1}{10 \delta_{r m s}} .
$$

If $\delta_{r m s}=6 \times 10^{-4}$, a value of $\alpha_{1}$ smaller than 170 is required, which is not necessarily easy to get since $\alpha_{0}$ itself is very small.

The above comments suggest that $\alpha_{1}$ is a very important lattice parameter which has to be controlled in the small $\eta$ regime. Next, we turn to establish the relation between the momentum compaction factor and the dispersion function, with the "wiggling effect" included.

\section{THE WIGGLING FACTOR}

To describe the closed orbit $x_{c o}(s, \delta)$ of an off-momentum particle, the dispersion function is introduced as

$$
D(s, \delta)=\frac{x_{c o}(s, \delta)-x_{c o}(s, 0)}{\delta}=D_{0}(s)+D_{1}(s) \delta+O\left(\delta^{2}\right),
$$

where $x_{c o}(s, 0)$ is the reference orbit, and $s$ is the azimuthal coordinate. Usually we refer to $D_{0}$, the linear part of dispersion function, as the dispersion function because $\delta \ll 1$. Since we are interested in $\alpha_{1}$, the first nonlinear part, $D_{1}$, has also to be included. Furthermore, the effect of closed orbit offset on $\alpha_{1}$ is negligible (Appendix A), therefore we can assume $x_{c o}(s, 0)=0$. 
Now, consider an infinitesimal piece of arc shown schematically in Fig. 1, where the orbit length of a synchronous particle $\left(\mathrm{AA}^{\prime}\right)$ is $d s=\rho d \theta$ of curvature. Then a particle with momentum offset $\delta$, will follow the orbit $\mathrm{BB}^{\prime}$ with length

$$
d l_{2}=d l_{1} \sqrt{1+\left(D_{0}^{\prime} \delta\right)^{2}}=d s\left[1+\frac{D_{0}}{\rho} \delta+\left(\frac{D_{1}}{\rho}+\frac{1}{2} D_{0}^{\prime 2}\right) \delta^{2}\right]
$$

rather than

$$
d l_{1}=\left(\rho+D_{0} \delta+D_{1} \delta^{2}\right) d \theta=d s\left[1+\frac{D_{0}}{\rho} \delta+\frac{D_{1}}{\rho} \delta^{2}\right],
$$

where $D_{0}^{\prime}=\frac{d D_{0}}{d s}$. Notice the difference between $d l_{2}$ and $d l_{1}$ in second order, which is called the "wiggling effect". This relation is also valid for a straight sector if the limit $\rho \rightarrow \infty$ is taken in the appropriate way. There, the only difference in orbit length is due to the "wiggling effect". The assumption has been made that there is no vertical dispersion.

The difference in total closed orbit length of an off-momentum particle from that of a reference particle is simply

$$
\Delta C=\oint\left(d l_{2}-d s\right)=\oint \alpha_{0}\left(\delta+\alpha_{1} \delta^{2}\right) d s
$$

Comparison of Equation (1) and Equation (12) yields

$$
\alpha_{0}=\frac{1}{C_{0}} \oint \frac{D_{0}}{\rho} d s \equiv\left\langle\frac{D_{0}}{\rho}\right\rangle
$$

and

$$
\alpha_{1}=\frac{\left\langle D_{1} / \rho\right\rangle}{\alpha_{0}}+\frac{\left\langle D_{0}^{\prime 2}\right\rangle}{2 \alpha_{0}}
$$

where $C_{0}=\oint d s,\langle\ldots\rangle$ means the average in the whole ring, and the last term in Equation (14) is called the wiggling factor: ${ }^{10}$

$$
w=\frac{1}{2 \alpha_{0}}\left\langle D_{0}^{\prime 2}\right\rangle=\frac{\left\langle D_{0}^{\prime 2}\right\rangle}{2\left\langle D_{0} / \rho\right\rangle} .
$$

This term is missing from some references, but will be shown to have significant contribution to $\alpha_{1}$.

Betatron oscillations may also contribute to the difference in orbit length. ${ }^{9,11}$ This is primarily, not a momentum dependent effect. In general, this effect is very small. 


\section{DIFFERENTIAL EQUATIONS FOR THE DISPERSION FUNCTION}

Motion in a circular accelerator is conveniently represented by the Hamiltonian with azimuthal coordinate $s$ instead of time $t$ as an independent variable

$$
\begin{aligned}
H=-p_{s} & =-\frac{e A_{s}}{c}-\left(1+\frac{x}{\rho}\right) p\left[1-\left(\frac{p_{x}}{p}\right)^{2}-\left(\frac{p_{y}}{p}\right)^{2}\right]^{1 / 2} \\
& \approx-\frac{e A_{s}}{c}-\left(1+\frac{x}{\rho}\right) p\left[1-\frac{1}{2}\left(\frac{p_{x}}{p}\right)^{2}-\frac{1}{2}\left(\frac{p_{y}}{p}\right)^{2}\right]
\end{aligned}
$$

where the canonical vector potential

$$
A_{s}=-\frac{p_{0} c}{e}\left[\frac{x}{\rho}+\left(\frac{1}{\rho^{2}}-K_{1}\right) \frac{x^{2}}{2}+\frac{K_{1} y^{2}}{2}+\frac{1}{6} K_{2}\left(x^{3}-3 x y^{2}\right)\right],
$$

for a lattice composed of separated-function magnets with hard edge. Here $K_{1}$ and $K_{2}$ are respectively the quadrupole and the sextupole strength for a reference particle

$$
K_{1}=\frac{e}{p_{0} c} \frac{\partial B_{y}}{\partial x}, \quad K_{2}=\frac{e}{p_{0} c} \frac{\partial^{2} B_{y}}{\partial x^{2}},
$$

The canonical differential equations of motion are obtained by partial differentiation of $H$, which expanded become

$$
\begin{aligned}
& x^{\prime}=\frac{\partial H}{\partial p_{x}}=\left(1+\frac{x}{\rho}\right) \frac{p_{x}}{p} \\
& p_{x}^{\prime}=-\frac{\partial H}{\partial x}=-p_{0}\left(\frac{1}{\rho^{2}}-K_{1}\right) x+\frac{p-p_{0}}{\rho}+\frac{p_{0}\left(x^{\prime 2}+y^{\prime 2}\right)}{2 \rho}-\frac{p_{0}}{2} K_{2}\left(x^{2}-y^{2}\right),
\end{aligned}
$$

where the prime represents the differentiation with respect to $s$, the azimuthal coordinate. Combining Equation (18) and Equation (19) we get the equation of motion

$$
x^{\prime \prime}+\left(1+\frac{x}{\rho}\right) \frac{1}{1+\delta}\left(\frac{1}{\rho^{2}}-K_{1}\right) x=\left(1+\frac{x}{\rho}\right) \frac{\delta}{1+\delta} \frac{1}{\rho}+\frac{x^{\prime} 2+y^{\prime 2}}{2 \rho}-\frac{1}{2} K_{2}\left(x^{2}-y^{2}\right),
$$

which is exact to order $\delta^{2}$. Substituting $x=D_{0} \delta+D_{1} \delta^{2}$ and $y=0$ yields

$$
\begin{aligned}
& D_{0}^{\prime \prime}+\left(\frac{1}{\rho^{2}}-K_{1}\right) D_{0}=\frac{1}{\rho} \\
& D_{1}^{\prime \prime}+\left(\frac{1}{\rho^{2}}-K_{1}\right) D_{1}=\frac{D_{0}^{\prime 2}}{2 \rho}-K_{1} D_{0}-\frac{1}{\rho}\left(1-\frac{D_{0}}{\rho}\right)^{2}-\frac{1}{2} K_{2} D_{0}^{2} .
\end{aligned}
$$




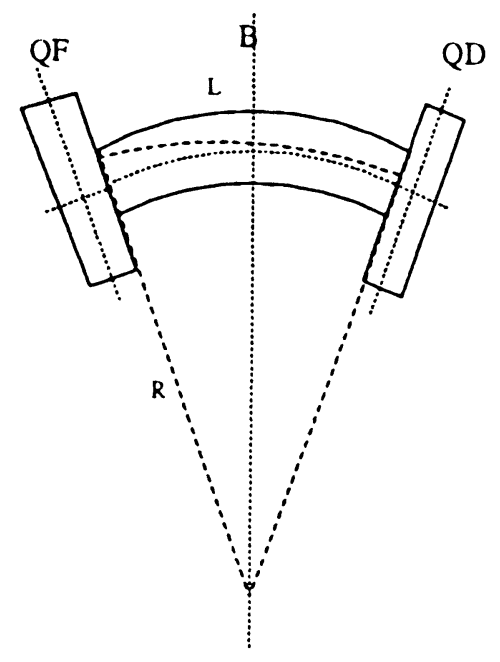

FIGURE 2: Schematic view of the half cell

These inhomogenous Hill equations could be solved in principle by using Green's function. ${ }^{12}$ But it is not obvious to see how the dispersion function is related to other lattice parameters. In the next section, we will solve Equation (21) and Equation (22) explicitly for an ideal FODO lattice. This is not far away from some realistic lattices, as shown later.

\section{A SOLUBLE CASE: THE IDEAL FODO LATTICE}

The ideal FODO lattice that we will consider is composed of $\mathrm{N}$ identical FODO cells, or $2 \mathrm{~N}$ half cells. Each half cell (Figure 2) starts at the center of a thin focussing quadrupole $(\mathrm{QF})$ and ends at the center of a neighboring thin defocussing quadrupole (QD). The absolute integrated strength of half QF and half QD is the same:

$$
q=\left|K_{1}\right| l_{h Q}=\frac{1}{f},
$$

where $K_{1}$ is the quadrupole gradient, and $l_{h Q}$ and $f$ are respectively the physical length and the focal length of the half quadrupole. The bending angle of each dipole is

$$
\theta_{0}=\frac{\pi}{N}=\frac{L}{R}
$$


where $\mathrm{L}$ is the half cell length, or the length of each dipole since $l_{h Q} \rightarrow 0$, and $\mathrm{R}$ is the radius of curvature for the reference particle. Another characteristic parameter is

$$
s=q L \approx \sin \phi_{1 / 2},
$$

which should not be confused with azimuthal coordinate $s$, where $\phi_{1 / 2}$ is the betatron phase advance per half cell. It seems that one only needs to solve Equation (21) and Equation (22) in the dipole. Actually, the necessary boundary conditions also have to be imposed by the thin quadrupoles.

In the dipole ( $\rho=R$ and $\left.K_{1}=0\right)$, Equation (21) reduces to

$$
D_{0 B}^{\prime \prime}+\frac{1}{R^{2}} D_{0 B}=\frac{1}{R}
$$

which is equivalent to

$$
\ddot{D}_{0 B}+D_{0 B}=R,
$$

where a dot represents the differentiation with respect to $\theta$. The general solution of Equation (25) is

$$
D_{0 B}(\theta)=R\left(1+c_{1} \sin \theta+c_{2} \cos \theta\right) .
$$

In a quadrupole, Equation (22) reduces to

$$
D_{0 Q}^{\prime \prime}-K_{1} D_{0 Q}=0,
$$

which provides the boundary conditions

$$
D_{0 Q}^{\prime}\left( \pm \frac{\theta_{0}}{2}\right)=-q D_{0 Q}\left( \pm \frac{\theta_{0}}{2}\right) .
$$

Here, use has been made of the symmetry condition $D_{0 Q}^{\prime}=0$ at the center of the quadrupoles.

The continuity of $D$ and $\frac{d D}{d l_{2}}$

$$
\frac{d D}{d l_{2}}=\left(1+\frac{x}{\rho}\right)^{-1} \frac{d D}{d s}=\left(1+\frac{x}{\rho}\right)^{-1} D^{\prime}
$$

yields

$$
\begin{aligned}
& \dot{D}_{0 B}\left( \pm \frac{\theta_{0}}{2}\right)=R D_{0 Q}^{\prime}\left( \pm \frac{\theta_{0}}{2}\right)=-Q D_{0 B}\left( \pm \frac{\theta_{0}}{2}\right) \\
& \dot{D}_{1 B}\left( \pm \frac{\theta_{0}}{2}\right)=R D_{1 Q}^{\prime}\left( \pm \frac{\theta_{0}}{2}\right)+D_{0}\left( \pm \frac{\theta_{0}}{2}\right) D_{0 Q}^{\prime}\left( \pm \frac{\theta_{0}}{2}\right)
\end{aligned}
$$

where 


$$
Q=R q=\frac{s}{\theta_{0}}=\frac{N s}{\pi}
$$

Substituting

$$
\dot{D}_{0 B}(\theta)=R\left(c_{1} \cos \theta-c_{2} \sin \theta\right)
$$

into Equation (30), we arrive, after some algebra, at the solution

$$
c_{1}=\frac{c_{2}}{Q}=-\frac{Q}{\left(1+Q^{2}\right) \cos \frac{\theta_{0}}{2}} .
$$

With the aid of Equation (34), Equation (26) and Equation (33) can be rewritten as

$$
\begin{aligned}
& D_{0 B}(\theta)=R\left[1+c_{1}(\sin \theta+Q \cos \theta)\right], \\
& \dot{D}_{0 B}(\theta)=R c_{1}(\cos \theta-Q \sin \theta) .
\end{aligned}
$$

Subtituting Equation (35) into Equation (13) and Equation (15) gives

$$
\alpha_{0}=\frac{1}{\theta_{0}} \int_{-\frac{\theta_{0}}{2}}^{+\frac{\theta_{0}}{2}} d \theta \frac{D_{0 B}}{R}=1+c_{2} \frac{2 \sin \frac{\theta_{0}}{2}}{\theta_{0}}=1-\frac{2 t Q^{2}}{\theta_{0}\left(1+Q^{2}\right)},
$$

where $t=\tan \left(\frac{1}{2} \theta_{0}\right)$, and the wiggling factor

$$
w=\frac{\left\langle D_{0}^{\prime 2}\right\rangle}{2 \alpha_{0}}=\frac{Q^{2}\left[\theta_{0}\left(1+t^{2}\right)\left(1+Q^{2}\right)+2 t\left(1-Q^{2}\right)\right]}{4\left(1+Q^{2}\right)\left[\theta_{0}\left(1+Q^{2}\right)-2 t Q^{2}\right]} .
$$

The relationships

$$
\sin \theta_{0}=\frac{2 t}{1+t^{2}}, \quad \cos \theta_{0}=\frac{1-t^{2}}{1+t^{2}}
$$

have been used to simplify the result.

Following the same procedure, we now solve $D_{1}$. In the dipole

$$
D_{1 B}^{\prime \prime}+\frac{1}{R^{2}} D_{1 B}=-\frac{1}{R}\left(\frac{D_{0 B}}{R}-1\right)^{2}+\frac{1}{R} \frac{D_{0 B}^{\prime 2}}{2}
$$

with the general solution

$$
\begin{aligned}
D_{1 B}(\theta)= & R\left[c_{3} \sin \theta+c_{4} \cos \theta\right] \\
& -R\left[\frac{1}{4} c_{1}^{2}\left(1+Q^{2}\right)+\frac{1}{4} c_{1}^{2}\left(1-Q^{2}\right) \cos 2 \theta-\frac{1}{2} Q c_{1}^{2} \sin 2 \theta\right] .
\end{aligned}
$$


Before solving the equation, notice

$$
D_{1 B}(\theta)=R\left[c_{3} \sin \theta+c_{4} \cos \theta\right]-\frac{1}{2 R} \dot{D}_{0 B}^{2},
$$

which leads to a very simple closed result

$$
\alpha_{0} \alpha_{1}=\frac{1}{\theta_{0}} \int_{-\frac{\theta_{0}}{2}}^{+\frac{\theta_{0}}{2}} d \theta\left(\frac{D_{1 B}}{R}+\frac{1}{2 R^{2}} \dot{D}_{0 B}^{2}\right)=c_{4} \frac{2 \sin \frac{\theta_{0}}{2}}{\theta_{0}} .
$$

To find $\alpha_{1}$, we only need to calculate $c_{4}$. Let's proceed with Equation (23) in the thin quadrupole

$$
D_{1 Q}^{\prime \prime}-K_{1} D_{1 Q}=-K_{1} D_{0 Q}
$$

which leads to

$$
D_{1 Q}^{\prime}\left( \pm \frac{\theta_{0}}{2}\right)=-q\left[D_{1 Q}\left( \pm \frac{\theta_{0}}{2}\right)-D_{0 Q}\left( \pm \frac{\theta_{0}}{2}\right)\right] .
$$

With Equation (31), the boundary conditions become

$$
\dot{D_{1 B}}\left( \pm \frac{\theta_{0}}{2}\right)=-Q\left[D_{1 B}\left( \pm \frac{\theta_{0}}{2}\right)-D_{0 B}\left( \pm \frac{\theta_{0}}{2}\right)+\frac{D_{0 B}^{2}\left( \pm \frac{\theta_{0}}{2}\right)}{R}\right] .
$$

By solving Equation (46), we find

$$
c_{4}=\frac{Q^{4}\left(Q^{2} t^{2}+3\right)}{2 \cos \frac{\theta_{0}}{2}\left(1+Q^{2}\right)^{3}} .
$$

Substituting Equation (47) into Equation (43) we have

$$
\alpha_{0} \alpha_{1}=\frac{Q^{4} t\left(Q^{2} t^{2}+3\right)}{\theta_{0}\left(1+Q^{2}\right)^{3}} .
$$

Further substitution of Equation (37) into Equation (48) allows one to write

$$
\alpha_{1}=\frac{Q^{4} t\left(Q^{2} t^{2}+3\right)}{\left(1+Q^{2}\right)^{2}\left[\theta_{0}\left(1+Q^{2}\right)-2 t Q^{2}\right]},
$$

which could alternatively be expressed as a function of $s$

$$
\alpha_{1}=\frac{s^{4} t\left(s^{2} t^{2}+3 \theta_{0}^{2}\right)}{\left(\theta_{0}^{2}+s^{2}\right)^{2}\left[\theta_{0}\left(\theta_{0}^{2}+s^{2}\right)-2 t s^{2}\right]} .
$$

Additionally, this result was independently reached through a geometric approach. ${ }^{13}$ Notice that both $w$ and $\alpha_{1}$ depend only on the strength of the quadrupoles and the number of cells. Figure 3 and Figure 4 are plots of $w$ and $\alpha_{1}$ respectively as a function of $s$ with a different number of cells. For a given $\mathbf{N}, w$ and $\alpha_{1}$ increase as 


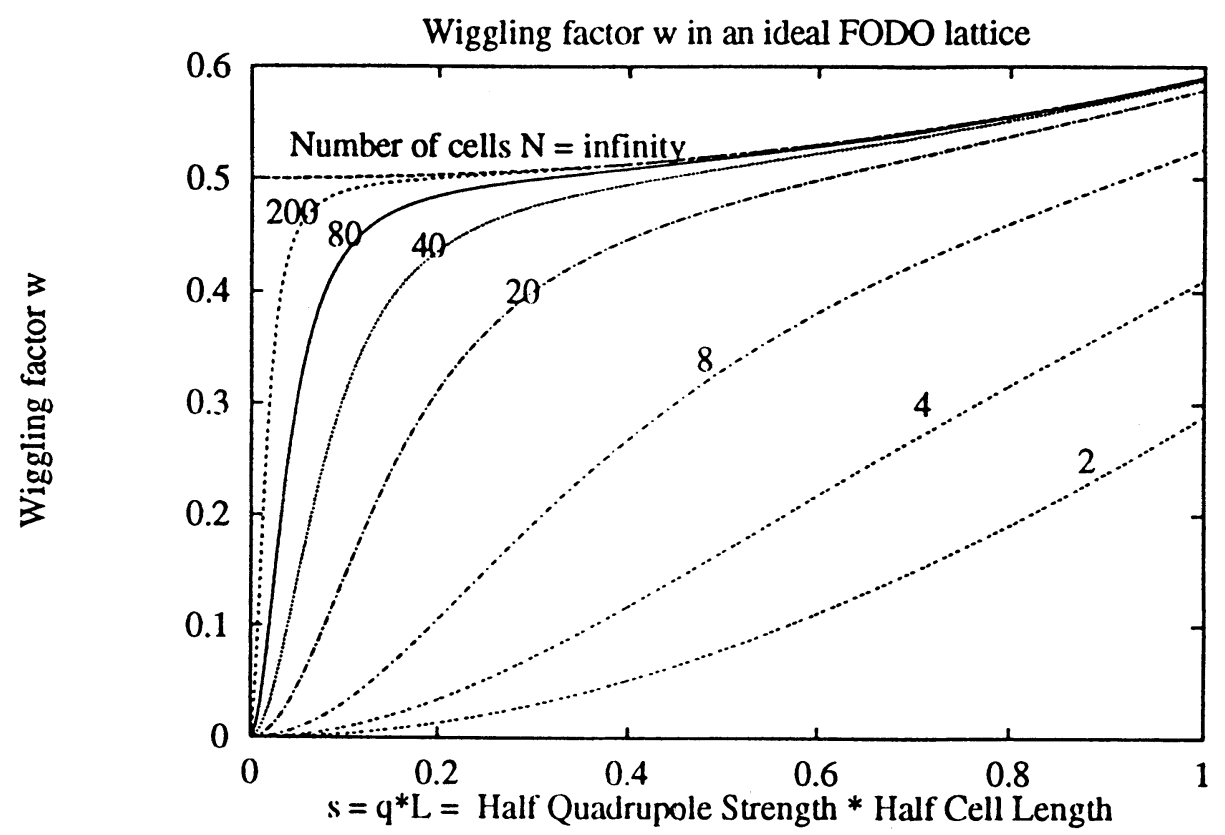

FIGURE 3: The wiggling factor $w$ as a function of $s\left(\approx \sin \phi_{\frac{1}{2}}\right)$ for a different number of cells in an ideal FODO lattice.

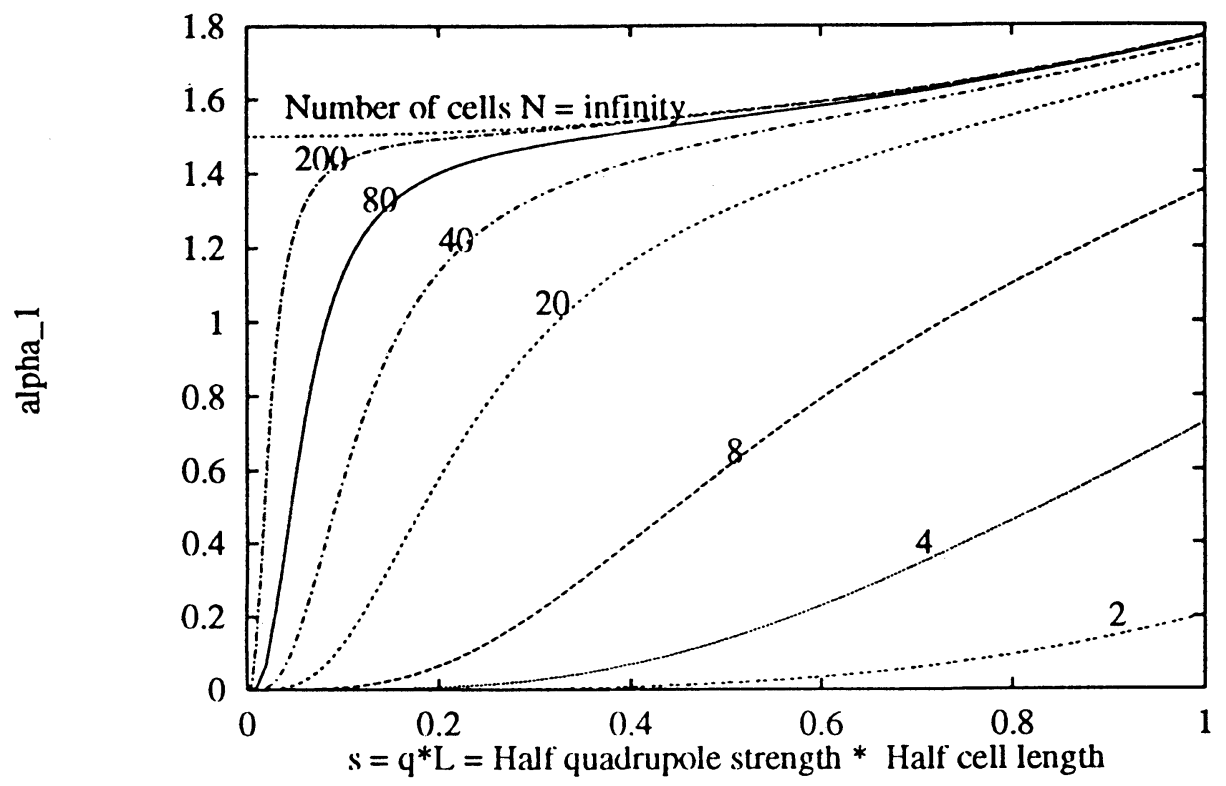

FIGURE 4: $\alpha_{1}$ as a function of $s\left(\approx \sin \phi_{\frac{1}{2}}\right)$ with a different number of cells in an ideal FODO lattice. 
quadrupoles become stronger. The possible value of $s$ is somewhere between 0 and 1 since $s \approx \sin \phi_{\frac{1}{2}}$. In the case $s=0$ (cyclotron), from Equation (37) and Equation (49) we have $\alpha_{0}=1$ and $\alpha_{1}=0$, as expected. We can show from symmetry that $\alpha_{n}(n \geq 1)=0$. For real synchrotrons, $\phi_{\frac{1}{2}}$ is usually between 30 and 45 degrees, and the operating range for $s$ is $0.5 \sim 0.7$. Notice that $w$ and $\alpha_{1}$ increase with $N$. Since $N$ increases as a factor of ring size (roughly $N \propto \sqrt{R}$ ), $w$ and $\alpha_{1}$ are bigger for larger machines.

In the case $N \rightarrow \infty$, the centrifugal focusing of dipoles becomes negligible, and the analytical results reduce to

$$
\begin{gathered}
\alpha_{0}=\frac{1}{Q^{2}}\left(1-\frac{s^{2}}{12}\right), \\
w=\frac{1+\frac{s^{2}}{12}}{2\left(1-\frac{s^{2}}{12}\right)}, \\
\alpha_{1}=\frac{3\left(1+\frac{s^{2}}{12}\right)}{2\left(1-\frac{s^{2}}{12}\right)}=3 w .
\end{gathered}
$$

\section{COMPARISON WITH MAD}

In general, the differential equations cannot be solved analytically and numerical method has to be used. Unfortunately, $\alpha_{1}$ is not directly available from the general codes such as MAD, ${ }^{14}$ which instead return the momentum compaction factor $\alpha_{p}$. The value of $\alpha_{1}$ has to be extracted from the dependence of $\alpha_{p}$ on $\delta$. Care must be taken about which definition of $\alpha_{p}$ is used in a specific code. It may be

$$
\alpha_{p 1}=\frac{p}{C} \frac{d C}{d p}=\alpha_{0}\left[1+2\left(\alpha_{1}+\frac{1}{2}-\frac{1}{2} \alpha_{0}\right) \delta\right]+O\left(\delta^{2}\right)
$$

or

$$
\alpha_{p 2}=\frac{p_{s}}{C_{s}} \frac{d C}{d p}=\alpha_{0}\left(1+2 \alpha_{1} \delta\right)+O\left(\delta^{2}\right),
$$

or something else. It is also important to test these codes using some very simple lattices, for which an analytical solution is possible. If there is good agreement, we can have confidence in numerical solutions of realistic lattices such as the Main Ring Main Injector, or an isochronous ring.

A lattice composed of 80 simplified FODO cells was set up as input to MAD. The length of a quadrupole was chosen as 1 micron. For every $s$, the momentum compaction factor, $\alpha_{p}$, is calculated by MAD at three momentum offsets, 




FIGURE 5: The comparison of $\alpha_{1}$ calculated from MAD (diamond symbols) and predicted by the analytical expression (solid line) for an ideal FODO lattice with 80 cells (sector dipoles). The difference between the sector dipole case (diamond symbols) and the rectangular dipole case (plus symbols) becomes important in weak focusing machines. Also, one can see when the approximate formula $(N=\infty$, dash line) begins to deviate from the exact result (solid line).

$\delta=-0.001,0,+0.001$. Then $\alpha_{1}$ was extracted from $\alpha_{p}$ depending upon the definition of $\alpha_{p}$, either

$$
\alpha_{1}=\frac{\alpha_{p 1}(\delta)-\alpha_{p 1}(-\delta)}{4 \alpha_{0} \delta}-\frac{1}{2}+\frac{\alpha_{0}}{2}
$$

if $\alpha_{p}=\alpha_{p 1}$, or

$$
\alpha_{1}=\frac{\alpha_{p 2}(\delta)-\alpha_{p 2}(-\delta)}{4 \alpha_{0} \delta},
$$

if $\alpha_{p}=\alpha_{p 2}$. If we use the first definition, a large disagreement occurs between the MAD result and the theoretical prediction from Equation (52). However, excellent agreement is achieved using the second definition, as shown in Figure 5. The systematic discrepancy found in Ref. 15 is now understood. 


\section{DEVIATION FROM THE IDEAL FODO LATTICE}

Taking the Main Injector $\left(N=80, s=\sin \frac{\pi}{4}=\frac{1}{\sqrt{2}}\right)^{16}$ as an example, we will see how the deviations from an ideal FODO lattice affect $\alpha_{1}$.

\subsection{Sector Dipoles and Rectangular Dipoles}

In the ideal lattice, we assumed there was no dipole edge focusing, as with sector dipoles. In reality the Main Injector dipole is rectangular. How important is this? Figure 5 shows that the difference between sector dipoles and rectangular dipoles is negligible in the case of Main Injector. But as the cell phase advance and/or number of cells decreases the edge focusing becomes more important. Therefore, special care has to be taken with edge focusing in small accelerator rings.

\subsection{Finite Length Quadrupole}

For the simplicity of analytical solution, we have used a thin quadrupole approximation. What happens if the quadrupole has a finite length? From the MAD calculation, Table 1, we see that the contribution of finite quadrupole length is also negligible. This is not a surprise because the dominant source of momentum compaction comes from dispersion in dipoles, and the boundary conditions are dominated by the integrated strength of the quadrupoles.

TABLE 1: The dependence of $\alpha_{1}$ on the half quadrupole length

\begin{tabular}{lccc}
\hline Half Quadrupole Length $l_{Q}(\mathrm{~m})$ & $1 \times 10^{-6}$ & 0.1 & 0.5 \\
\hline$\alpha_{1}$ & 1.545 & 1.546 & 1.550 \\
\hline
\end{tabular}

\subsection{Contribution from Sextupoles}

Because of the head-tail instability, the natural chromaticity is usually compensated by sextupoles. If the sextupole strengths are set to make the net chromaticity $1-f$ times the natural chromaticity, $D_{0}$ (and thus $\alpha_{0}$ and $w$ ) will not change while $D_{1}$ will be modified as shown, in ${ }^{15}$

$$
\left\langle D_{1}\right\rangle \approx \frac{R}{Q^{2}}\left(1-f+\frac{s^{2}}{12}\right) .
$$

This approximation is true when the focussing from dipoles is negligible. Then

$$
\alpha_{1}=\frac{3-2 f+\frac{s^{2}}{4}}{2\left(1-\frac{s^{2}}{12}\right)} .
$$


When the net chromaticity is compensated to zero, or $f=1$,

$$
0.5 \leq \alpha_{1}=\frac{1+\frac{s^{2}}{4}}{2\left(1-\frac{s^{2}}{12}\right)} \leq .68,
$$

because $0 \leq s \leq 1$. For the Main Injector $s^{2}=0.5$, and we have $\alpha_{1}=0.587$. A value of $\alpha_{1}=-1.5$ can be obtained in principle by setting

$$
f \approx 3,
$$

resulting in desirably strong nonlinear fields.

\section{CONCLUSION AND DISCUSSION}

Starting with a Hamiltonian, we derived the differential equation for the two leading terms in the dispersion function, which is exact for any lattice composed of separatedfunction magnets with hard edge. The linear term $D_{0}$ is determined only by linear elements (dipoles and quadrupoles). The first nonlinear correction $D_{1}$ also depends on sextupoles, but not on octupoles or higher order magnets.

For an ideal FODO lattice, the differential equations were solved to get analytical expressions for $\alpha_{0}, w$, and $\alpha_{1}$. A comparison with MAD calculations of momentum compaction factor showed perfect agreement. MAD was then used to show that conventional FODO-like lattices are not far away from the ideal one. In a large machine such as the Fermilab Main Injector, we found that $\alpha_{1}$ is not sensitive to quadrupole length and edge focusing.

\section{ACKNOWLEDGEMENTS}

The possible importance of the previously neglected wiggling factor was first pointed out to us by Leo Michelotti. We thank K.Y. Ng for his help in the discussion of differential equations. Discussions with W. Gabella were also very fruitful. Thanks also go to C. Ankenbrandt for his comments.

\section{REFERENCES}

1. K. Johnsen, in Proceeding of the CERN Symposium on High Energy Accelerators and Pion Physics, CERN, 1956, pp.106-109.

2. J. Wei, Longitudinal Dynamics of the Non-Adiabatic Regime on Alternating-Gradient Synchrotons, Ph.D. dissertation, State University of New York at Stony Brook, May 1990.

3. S.A. Bogacz, in Proceedings of the Fermilab III Instabilities Workshop, (Fermilab, June 1990), p.116.

4. I. Kourbanis and K.Y. Ng, in Proceedings of the Fermilab III Instabilities Workshop, (Fermilab, June 1990), pp.141-150.

5. I. Kourbanis, K. Meisner, and K.Y. Ng, in Proceedings of the IEEE Particle Accelarators Conference (San Francisco, 1991).

6. D.A.G. Deacon, Physics Report, 76, 5 (1981) 349-391. 
7. S. Chattopadhyay et al., in Proceedings of ICFA Workshop on Low Emittance $e^{-} e^{+}$beams, Brookhaven National Laboratory report BNL-52090 (1987).

8. C. Pellegrini and D. Robin, Nucl. Instrum. Meth. A301 (1991) 27-36.

9. H. Bruck et al., IEEE Trans. Nucl. Sci., 20(3):822, June 1973.

10. E. Ciapala, A. Hofmann, S. Myers, and T. Risselada, IEEE Trans. Nucl. Sci., 26(3), June 1979.

11. L. Emery, in Proceedings of the 15th International Conference of High Energy Accelerators, p.1172, Hamburg, 1992.

12. J.-P. Delahaye and J. Jager, Particle Accelerators, 18:183-201, 1986.

13. K.Y. Ng, Fermilab report FN 578 (December 1991).

14. H. Grote and F.C. Iselin, The MAD Program (Methodical Accelerator Design), User's Reference Manual. CERN, 8.1 edition (September 1990).

15. S.A. Bogacz and S.G. Peggs, in Proceedings of the Fermilab III Instabilities Workshop, pp.192-206, Fermilab, June 1990.

16. Fermilab Main Injector, Conceptual Design Report, Revision 2.3, appendum, Fermilab (March 1991). 


\section{APPENDIX A. THE EFFECT OF CLOSED ORBIT OFFSET ON $\alpha_{1}$}

In section 2 we assume that closed reference orbit is flat, $x_{c o}=0$. Actually there is always a closed orbit offset due to various errors. Since such an offset, $x_{c o}$, is comparable to $D \delta$, it is natural to ask if it will affect the calculation of $\alpha_{1}$. The answer is that this effect is negligible for any realistic orbit offset.

For mathematical convenience, consider a simple model lattice, where the undistorted closed orbit is a circle with radius $R$. If the distorted closed orbit for a reference particle is $x_{c o}(s)$, its circumference will be

$$
C_{0}=2 \pi R\left(1+\frac{\left\langle x_{c o}\right\rangle}{R}+\frac{1}{2}\left\langle x_{c o}^{\prime 2}\right\rangle\right)
$$

where $\langle x\rangle=\frac{1}{2 \pi R} \oint x d s$. The closed orbit length of a particle with momentum offset $\delta$ is

$$
C=2 \pi R\left[1+\frac{\left\langle x_{c o}(\delta)\right\rangle}{R}+\frac{1}{2}\left\langle x_{c o}^{\prime 2}(\delta)\right\rangle\right]
$$

where

$$
x(\delta)_{c 0}=x_{c o}+D_{0} \delta+D_{1} \delta^{2} .
$$

If Equation (63) is reorganized into an expansion of $\delta$ with the aid of Equation (62) and compared to Equation (1), we get

$$
\alpha_{0}=\frac{\frac{\left\langle D_{0}\right\rangle}{R}+\left\langle x_{c o}^{\prime} D_{0}^{\prime}\right\rangle}{1+\frac{\left\langle x_{c o}\right\rangle}{R}+\frac{1}{2}\left\langle x_{c o}^{\prime 2}\right\rangle}
$$

and

$$
\alpha_{1}=\frac{\frac{\left\langle D_{1}\right\rangle}{R}+\frac{1}{2}\left\langle D_{0}^{\prime 2}\right\rangle+\left\langle x_{c o}^{\prime} D_{1}^{\prime}\right\rangle}{\frac{\left\langle D_{0}\right\rangle}{R}+\left\langle x_{c o}^{\prime} D_{0}^{\prime}\right\rangle} .
$$

Before orbit correction, the orbit offset wave has approximately the same wavelength (cell length) as the dispersion wave. The effect of closed orbit offset on $\alpha_{1}$ is negligible so long as $x_{c o} \ll D_{0}, D_{1}$, which is always true in any practical case. After orbit correction the correlation between the orbit offset and the dispersion wave becomes much weaker, $\left\langle x_{c o}^{\prime} D_{0}^{\prime}\right\rangle$ and $\left\langle x_{c o}^{\prime} D_{1}^{\prime}\right\rangle$ average out to zero, and the condition $x_{c o} \ll$ $D_{0}, D_{1}$ may not be necessary. 